\title{
Primary care management of anaphylaxis
}

\author{
Sukhmeet Singh Panesar, Samantha Walker, Aziz Sheikh
}

\begin{abstract}
Introduction
Anaphylaxis is severe systemic allergic reaction, which is potentially life threatening.

It is estimated that there are between 20-30 deaths from anaphylaxis in the UK each year, many of which are potentially preventable. ${ }^{1}$ This figure is however likely to represent something of an underestimate because deaths from drug allergy and a proportion of deaths in those with a history of asthma and unexpected deaths may have been misclassified.
\end{abstract}

There are two main mechanisms involved, namely anaphylactic (IgE-mediated) and anaphylactoid (pseudoallergic non IgE-mediated) reactions. In both anaphylactic and anaphylactoid reactions, a very similar clinical picture develops requiring identical immediate management; the precise mechanism involved may however be important in guiding investigations in order to identify possible trigger(s) as IgE-mediated disease is amenable to investigation using skin-prick testing and measurements of serum-specific IgE. ${ }^{2}$ Serum tryptase measurements taken during the acute episode can help differentiate between anaphylactic and anaphylactoid reactions and should if possible be taken by the attending GP.

\section{Clinical features}

As there is no uniformly accepted objective definition for anaphylaxis, ${ }^{3}$ diagnosis depends on the identification of a constellation of symptoms and signs, which may involve a number of organ systems:

- Skin: erythema, pruritis, urticaria and angioedema

- Eyes: injection of conjunctiva, tearing and itching

- Airways: sneezing, rhinorrhoea, laryngeal oedema, cough, wheeze and dyspnoea

- Gastrointestinal: tongue swelling, abdominal cramps, vomiting and diarrhoea

- Cardiovascular: palpitations, light-headedness and collapse.

Clinical features may differ according to the type of exposure i.e. whether the offending substance has been inhaled, ingested or injected. Patients who experience iatrogenic- or venom-induced anaphylaxis are more likely to experience cardiovascular symptoms, whereas food exposure is more likely to result in respiratory symptoms. It should be recognised, however, that anaphylaxis is a dynamic event during which multiple symptoms may come and go but which, once recognised, must be promptly treated to prevent further development of systemic features. ${ }^{4}$

A working diagnosis of anaphylaxis can usually be made by identifying features of a systemic allergic reaction accompanied by either respiratory difficulty or features suggestive of hypotension. ${ }^{5}$

\section{Treatment}

Systematic reviews of evidence and randomised controlled trials are the gold standard study design for evaluating the effectiveness of interventions. But in the case of uncommon conditions and moreover those that are acute in onset and potentially life threatening, controlled intervention studies are extremely difficult to mount. Such limitations apply to the study of anaphylaxis and it is therefore necessary to base treatment decisions on other sources of evidence such as observational studies, expert opinion and personal experience. Notwithstanding their limitations, consensus statements for the management of anaphylaxis represent the best available summary of treatment approaches for the immediate treatment of anaphylaxis. ${ }^{6}$

The overall management of anaphylaxis may conveniently be divided into two stages: acute and longer-term care.

\section{Acute management}

The priority when managing acute attacks is to institute life-saving measures if required and to administer adrenaline at the earliest opportunity in order to abort the developing systemic reaction. Outlined below is a step-wise approach to acute management:

- Commence basic and advanced life support (if indicated)

- Oxygen

- Adrenaline (epinephrine) 1:1000 solution (IM) if not already administered (many patients with a history of anaphylaxis will have been issued with adrenaline for self-injection)

- Inhaled $\beta$-2-agonist if evidence of bronchospasm

- Repeat adrenaline five minutes after first dose if there is no clinical improvement

- Chlorpheniramine IM (or by slow IV injection)

- Crystalloid fluid infusion if symptoms of hypotension persist (a repeat dose may be necessary)

- Hydrocortisone IM (or by slow IV injection). ${ }^{7}$

\section{Longer-term management}

Identification of trigger agents is crucial to the longerterm care of anaphylaxis patients. A detailed clinical history is the most important part of the diagnostic process and should aim to identify possible triggers. Selective sensitivity testing with objective allergy tests - in the form of skin prick testing, serum-specific IgE testing and occasionally challenge testing - has an important role to play in identifying triggers. Skin

\section{Sukhmeet Singh}

Panesar

Imperial College, London

Samantha Walker

National Respiratory

Training Centre

\section{Aziz Sheikh}

University of Edinburgh

Correspondence to: Professor Aziz Sheikh Division of Community Health Sciences: General Practice Section University of Edinburgh 20, West Richmond St Edinburgh EH8 9DX

Tel: +44 (0)131 6514151

Fax: +44 (0)131 6509119

Email:

aziz.sheikh@ed.ac.uk

Date submitted: 20/10/03

Date accepted: 17/11/03

Prim Care Resp J 2003; 12(4):124-126 


\section{Useful patient and professional contacts}

\section{For patients}

\section{Anaphylaxis Campaign}

PO Box 275, Farnborough, Hampshire GU14 6SX

Tel: 01252542029

Fax: 01252377140

Website: www.anaphylaxis.org.uk

\section{British Allergy Foundation}

Deepdene House, 30 Bellegrove Road, Welling, Kent, DA16 3BY

Tel: 02083038583

Fax: 02083038792

Website: www.allergyfoundation.com

\section{MedicAlert Foundation}

17 Bridge Wharf, 156 Caledonian Road, London N1 9UU

Tel: 02078333034

Fax: 02077135653

Website: www.medicalert.co.uk/Default.htm

\section{For professionals}

British Society for Allergy \& Clinical Immunology 66 Weston Park, Thames Ditton, Surrey KT7 0HI

Tel: 02083989240

Fax: 02083982766

Website: www.soton.ac.uk/ bsaci

National Respiratory Training Centre

The Athenaeum, 10 Church Street, Warwick

CV34 4AB

Tel: 01926493313

Fax: 01926493224

Website: www.nart.org.uk

The American Academy of Allergy and Immunology

611 East Wells Street

Milwaukee, WI 53202

Tel: (414) 272-6071

Patient Information and Physician Referral Line

Tel: +01-1-800-822-2762

www.aaaai.org prick testing to anything other than aeroallergens is not recommended in general practice because of the risk of systemic reactions. Of these tests then, serum-specific IgE testing to foods, venom and latex is most conveniently undertaken in a primary care setting although specialist referral to an allergist (or general physician with an interest in anaphylaxis) of all patients who have experienced a generalised reaction is a key step to ensuring that patients are appropriately investigated and advised on ways of minimising future risk.

This increased convenience however needs to be counterbalanced with the knowledge that serum specific IgE is of low sensitivity compared to skin prick testing for many allergens including latex, many foods (including fruits and nuts) and drugs when deciding which diagnostic tests to request.

Familiarity with some of the most commonly encountered triggers is important both for identifying possible triggers and also for advising patients on ways of minimising further exposure. Foods (peanuts, other legumes, tree nuts (i.e. walnuts, pecans and others), fish, shellfish, milk and eggs and condiments), drugs (avian based vaccines, beta-lactam antibiotics, aspirin and other non-steroidal antiinflammatory drugs) and venom (insect stings and bites) represent the most common trigger agents although any of a number of other factors including occupational agents (such as latex), physical triggers (such as exercise) and bodily fluids (such as blood and seminal fluid) may also be responsible. Other more rare triggers include radiographic contrast material, insulin, protamine, local anaesthetics, progesterone and fluorescein. ${ }^{2}$

Other important aspects of the successful longer-term management of anaphylaxis that need routinely to be considered include:
- Ensure that the diagnosis and trigger agent(s) are clearly documented in hospital and general practitioner records (paper and electronic)

- Ensure that patient/carer is aware of diagnosis, trigger(s) and ways of minimising risk of reexposure

- Avoid use of non-cardiac selective $\beta$-blockers in those with a history of anaphylaxis

- Optimise asthma control in those with co-existing asthma

- Issue of self-administered adrenaline and training of patient/carer in its use

- Consider immunotherapy in those with history of venom induced anaphylaxis

- Recommend obtaining Medic Alert bracelet/necklace.

\section{Conclusions}

Evidence suggests that the UK incidence of anaphylaxis is increasing; possible reasons for this increase include a generalised increased susceptibility to allergic conditions and/or increased allergen exposure. ${ }^{8,9}$ Awareness of the early clinical features of anaphylaxis is important to allow prompt diagnosis and treatment with adrenaline as if left untreated death may occur within minutes. Experience suggests that the longer-term needs of these patients are frequently overlooked, this contributing to the risk of recurrence. Specialist assessment is important to allow identification of possible trigger factors and to consider the appropriateness and relative strengths of different approaches to longer-term care. Issue of, and training in use of, self-administered adrenaline is essential in those with a previous history of severe systemic allergic reactions but this need not necessarily be the optimal management approach in those with non life-threatening reactions because of the small but known risk of adverse events if incorrectly used. Desensitisation is an additional effective treatment approach in those with venomprovoked disease.

It needs to be remembered that living with anaphylaxis is for many people an extremely stressful experience. Anxieties and concerns, especially in the case of children, are likely to be high and one of the key roles of primary care professionals is to explore and tackle such issues aiming to assist those prone to anaphylaxis to live with their condition in confidence and a sense of self-control. ${ }^{10,11}$

\section{References}

1. Pumphrey RS. Lessons for management of anaphylaxis from a study of fatal reactions. Clin Exp Allergy 2000;308:1144-50.

2. Walker S and Sheikh A. Managing anaphylaxis effective emergency and long-term care are necessary. Clin Exp Allergy 2003;33:1015-8.

3. Project team of the Resuscitation Council (UK). Emergency medical treatment of anaphylactic reactions. J Accid Emerg Med 1999;16:243-48. 4. Pumphrey RS. Lessons for management of anaphylaxis from a study of fatal reactions. Clin Exp 


\section{Not to be reproduced without the permission of the Primary Care Respiratory Journal}

Allergy 2000;30:1144-50.

5. Ewan PW. Anaphylaxis. BMJ 1998;316:1442-45.

6. Project team of the Resuscitation Council (UK).

Update on the emergency medical treatment of anaphylaxis reactions for first medical responders and for community nurses. Resuscitation 2001:48:241-43.

7. Sheikh A. Anaphylaxis. Update 1999;58:984-88.

8. Sheikh A, Alves B. Hospital admissions for acute anaphylaxis: time trend study. BMJ 2000;320:1441.
9. Gupta R, Sheikh A, Strachan D, Anderson HR. Increasing hospital admissions for systemic allergic disorders in England: analysis of national admissions data. BMJ 2003;327:1142-3.

10. Mandell D, Curtis R, Gold M, Hardie S. Families coping with a diagnosis of anaphylaxis in a child: a qualitative study of informational and support needs. ACI International 2002;14:96-101.

11. Sheikh A. Allergic problems. In: Khot A, Polemar A (eds.). Practical general practice (4th ed). Oxford: Butterworth Heneiman, 2003:385-387.

\section{Call for Membership to GPIAG Committee}

I

$\mathrm{f}$ you are interested in becoming a more active member of the GPIAG, why not consider standing for election to the GPIAG committee?

The committee meet three times per year and regularly contribute to discussions and activities as well as being part of an email network.

This is an excellent way to be involved in setting the agenda for the management of respiratory disease in Primary Care at a national level. The committee acts as an advisory body to the Trustees, and its members invariably develop a national profile. If you have a burning desire to set the world of primary care respiratory disease management alight, then this is the post for you!

The time commitment is probably about one session a week, but this can vary depending on subcommittee commitments etc.

If you have a few hours per week to spare and would like to stand for election for GPIAG Committee membership please contact the GPIAG Secretariat on 01214548219 for a job description and details of how to apply. 